\title{
EXPERIMENTAL STUDY ON DETECTION OF REBAR CORROSION IN CONCRETE BASED ON METAL MAGNETIC MEMORY
}

\author{
Hong Zhang,, Jianting Zhou, ${ }^{*}$ Ruiqiang Zhao, ${ }^{* *}$ Leng Liao, ${ }^{* *}$ Mao Yang, ${ }^{* * *}$ and Runchuan Xia*
}

\begin{abstract}
This paper presents a non-destructive testing method for detection and analysis of rebar corrosion in concrete based on metal magnetic memory. A three-dimensional (3D) automatic scanning and measuring system, composed of a 3D scanning and measuring device and an automation software, was self-designed and developed. Two corroded reinforced concrete specimens were prepared by the accelerated galvanic corrosion test. A series of experiments were implemented by the proposed 3D device. The experimental processes involve acquiring the spatial position and corresponding $3 \mathrm{D}$ magnetic flux density near the specimens, judging the rebar's corroded region, and assessing the corrosion degree of the specimens. The results indicate that the curves of tangential magnetic field, obtained by the $Y$-scanning, all intersect near the edge of the steel corrosion zone, and the corrosion region can be qualitatively determined by the position and distance of the two intersecting points; the curves of tangential magnetic field, obtained by the $Z$-scanning, have an extreme value varying with the lift-off heights (LFHs) of the magnetic sensor in the corroded region, and the corrosion degree can be semi-quantitatively assessed by the LFH of the reversal point. The findings of this work propose an effective non-destructive detection method for rebar corrosion in concrete.
\end{abstract}

\section{Key Words}

Metal magnetic memory, rebar corrosion, non-destructive testing, $3 \mathrm{D}$ scanning and measuring

\section{Introduction}

Corrosion of steel rebar is one of the major diseases leading to performance deterioration and even the destruction of reinforced concrete $(\mathrm{RC})$ structures [1], which reduces the

* College of Civil Engineering, Chongqing Jiaotong University, Chongqing, People's Republic of China; e-mail: \{hongzhang, jtzhou\}@cqjtu.edu.cn, rcxia@mails.cqjtu.edu.cn

** School of Materials Science and Engineering, Chongqing Jiaotong University, Chongqing, People's Republic of China; e-mail: \{rqzhao, lengliao\}@cqjtu.edu.cn

*** College of Civil Engineering, Chongqing Three Gorges University, Chongqing, People's Republic of China; e-mail: yangmao199104@163.com

Recommended by Prof. Chaomin Luo

(DOI: 10.2316/Journal.206.2017.5.206-5082) durability and service life of RC structures [2]. Therefore, it is of great significance to detect corrosion in the $\mathrm{RC}$ structures, and timely take the reinforcement measures to the structures with corrosion diseases [3].

Currently, there are many methods to nondestructively test the rebar corrosion in the analytical [4], physical [5] and electrochemical [6] ways. The analytical methods work by measuring some key parameters and then speculating the corrosion degree according to some prediction models and the field environment. The parameters include the diameter of rebar, the thickness of the concrete cover, the strength of concrete, immersion depth of various harmful ions and the width of the longitudinal cracks [7]. However, the data analysis extremely depends on the reliable prediction models, while there are not reliable prediction models for rebar corrosion [4]. The physical methods are mainly used to assess the corrosion condition of rebar by measuring the physical parameters accompanying with corrosion, such as resistance, electromagnetic property, heat conduction and acoustic wave propagation. The physics-based measuring methods include the resistance probe method [8], the eddy current detection method [9], the X-ray method [10], the infrared thermography [11] and the acoustic emission detection techniques [12]. However, those physical methods are not completely applicable to the RC structures for the interference of the concrete. The electrochemical methods include the half-cell potential method, the linear polarization method and the electrochemical impedance spectroscopy method [6]. The half-cell potential method is widely used for the qualitative detection of rebar corrosion, but this method needs pre-treatments to the structure surface. The linear polarization method and the electrochemical impedance spectroscopy method can be used for quantitative detection of rebar corrosion. However, on the one hand, the former cannot judge the early corrosion, and the field operation is complex. On the other hand, the latter cannot reach as convenient and accurate as the former, and analysing the measuring data is sophisticated. Consequently, the electrochemical impedance spectroscopy method is not applicable to the field detection [13].

The non-destructive testing (NDT) technology based on metal magnetic memory (MMM) theory was first 


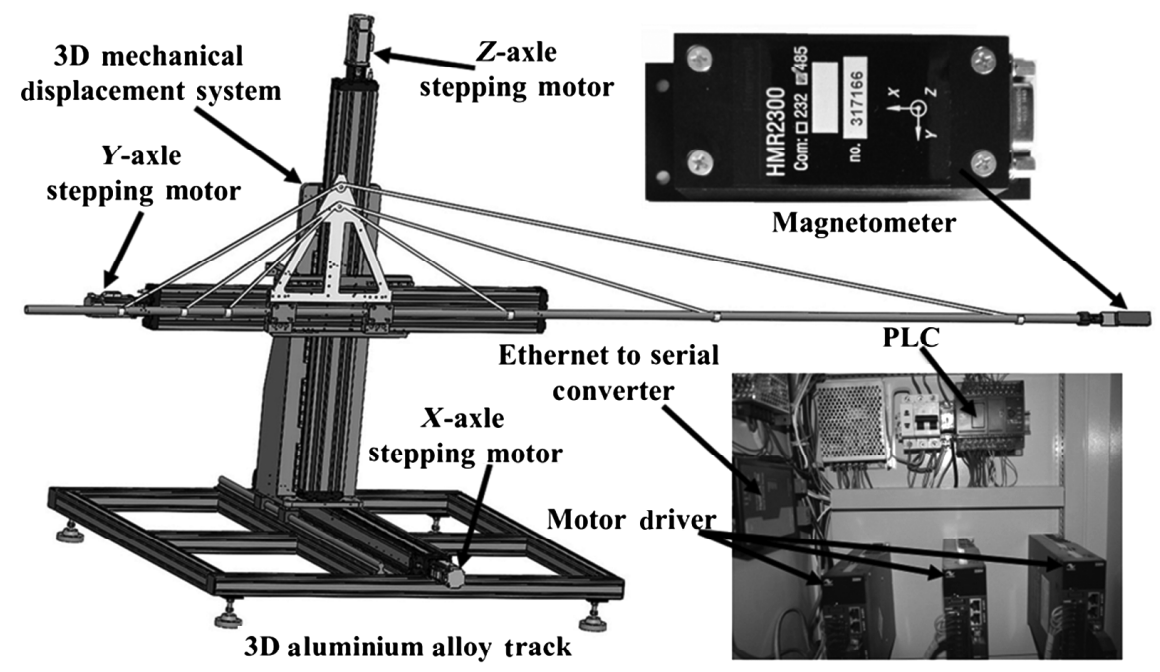

Figure 1. The structure graph of the 3D scanning and measuring device.

proposed by Dubov in the 1990s [14]. The fundamental principle of MMM is that the stress concentration (SC) and damaged regions of ferromagnetic materials produce selfmagnetic flux leakage (SMFL) signals due to the excitation of the Earth's magnetic field and mechanical loads, and the SMFL signals are irreversible after releasing the loads. MMM is a passive technique to detect the SMFL from the failure, which does not apply an artificial magnetic field as the stimulus source. It is known that the Earth's magnetic field is very weak (about $40 \mathrm{~A} / \mathrm{m}$ ), so the MMM technique is a weak-field test method. The information of structural damage is delivered by the SMFL signal, which is acquired by the high-precision micro-magnetic sensor [5], [15]. Unlike conventional NDT methods, the MMM technique can find early defects induced by high SC of steel structures. The method does not need any pre-treatment of structure surface, and it can detect defects not only on the surface but also inside structures [16]. The MMM method has got considerable attention for its advantages of time saving and easy operation [17], and it has been applied in the damage detections of pipelines, boilers, gas containers, steel wire ropes and welding cracks [18]. Meanwhile, the steel rebar is a kind of ferromagnetic material, and the corrosion of rebar in $\mathrm{RC}$ are often accompanied with stress concentration, so it is also a feasible method to detect the rebar corrosion based on the MMM technology.

In this paper, the MMM method is applied to detect the rebar corrosion in concrete with the assist of a selfdesigned three-dimensional (3D) automatic scanning and measuring equipment. The results indicate that the proposed method can qualitatively detect the corrosion position and semi-quantitatively evaluate the corrosion degree in RC structures.

\section{Design of 3D Automatic Scanning and Measuring System}

The SMFL signal of the ferromagnet is weak, and it is sensitive to the location of measuring sensor, so it is necessary to build an accurate spatial position measurement system, which can carry the sensor and scan automatically. To investigate the relationship between the SMFL signal and the degree of rebar corrosion, a 3D automatic scanning and measuring system [18], [19], composed of a 3D scanning and measuring device and an automation software system, is designed and built.

\subsection{D Scanning and Measuring Device}

Figure 1 shows the self-designed 3D scanning and measuring device for acquiring the 3D magnetic flux density and its corresponding spatial position around a specimen. The device consists of a 3D mechanical displacement system, a high-precision micro-magnetic sensor and an ethernet to serial converter (ESC) which owns two RS485 serial ports and one ethernet port.

The 3D mechanical displacement system is made up of a 3D aluminium alloy track and bracket system, three stepping motors and their corresponding motor drivers, a programmable logic controller (PLC) system and a nonmagnetic stainless steel pipe to support the sensor. Each motor driver can receive the PLC's commands and return the stepping motor's current displacement. The displacement accuracy of every stepping motor driver is as high as $0.1 \mathrm{~mm}$. A cable-stayed-like structure is used in the scanning device to improve the stability of the supporting bar when the device is moving. The Honeywell HMR2300 magnetometer is employed as the micro-magnetic sensor. It is a three-axis smart digital magnetometer, and the three axes oriented in orthogonal directions of HMR2300 magnetometer can measure the $X, Y$ and $Z$ vector components of magnetic flux density. The output range of HMR2300 magnetometer is \pm 2 Gs with a resolution of $\sim 70 \mu \mathrm{Gs}$. The PLC and HMR2300 magnetometer both have one RS485 serial data interface, and they can independently connect with the ESC via two disparate RS485 serial buses. The ESC can intelligently convert network data and serial data to each other. Thus, the proposed 3D scanning and measuring device can synchronously output the information of magnetic flux density $\left(\boldsymbol{B}_{x}, \boldsymbol{B}_{\boldsymbol{y}}\right.$ and $\left.\boldsymbol{B}_{\boldsymbol{z}}\right)$ and spatial 


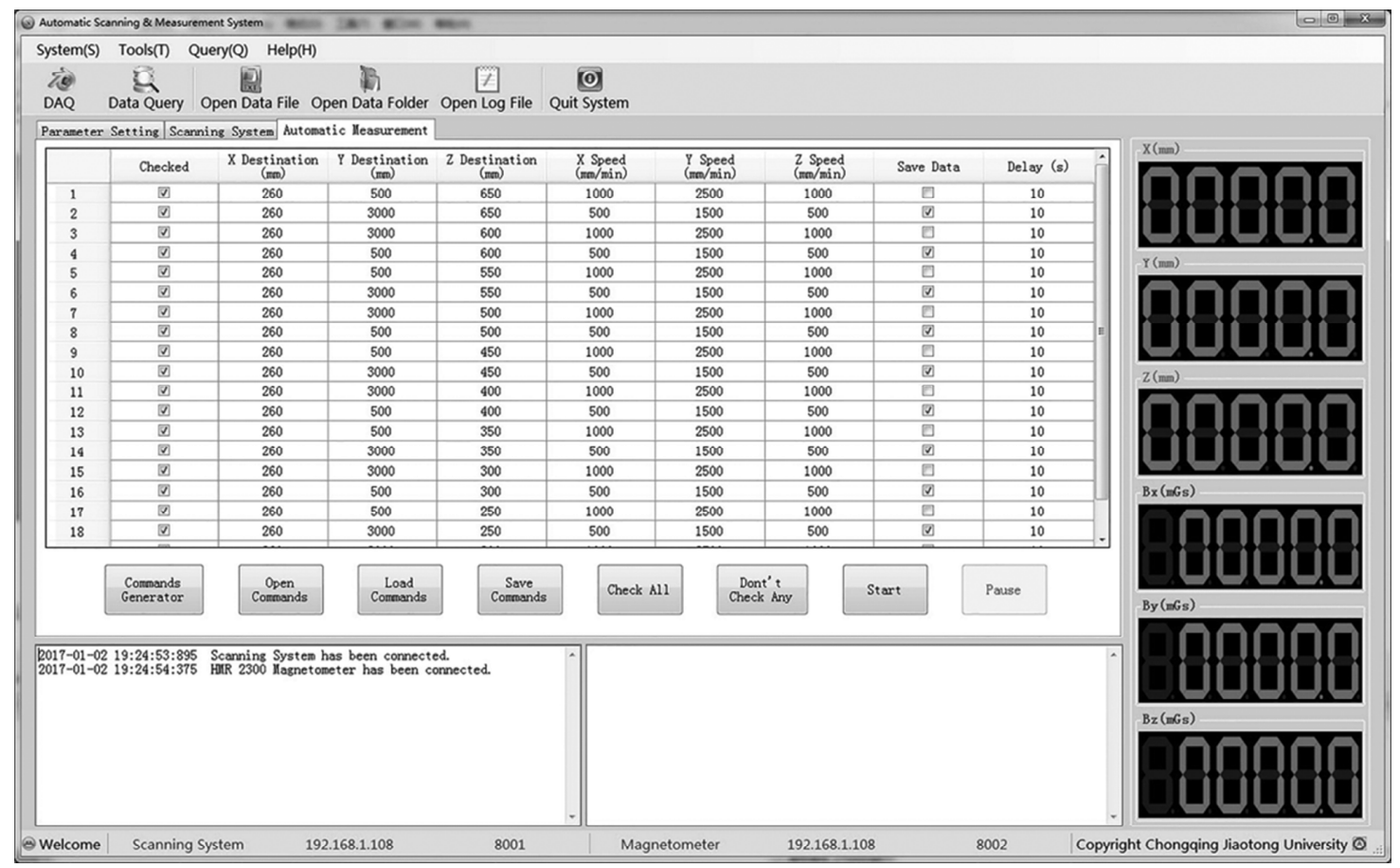

Figure 2. The interface of the scanning and measuring software.

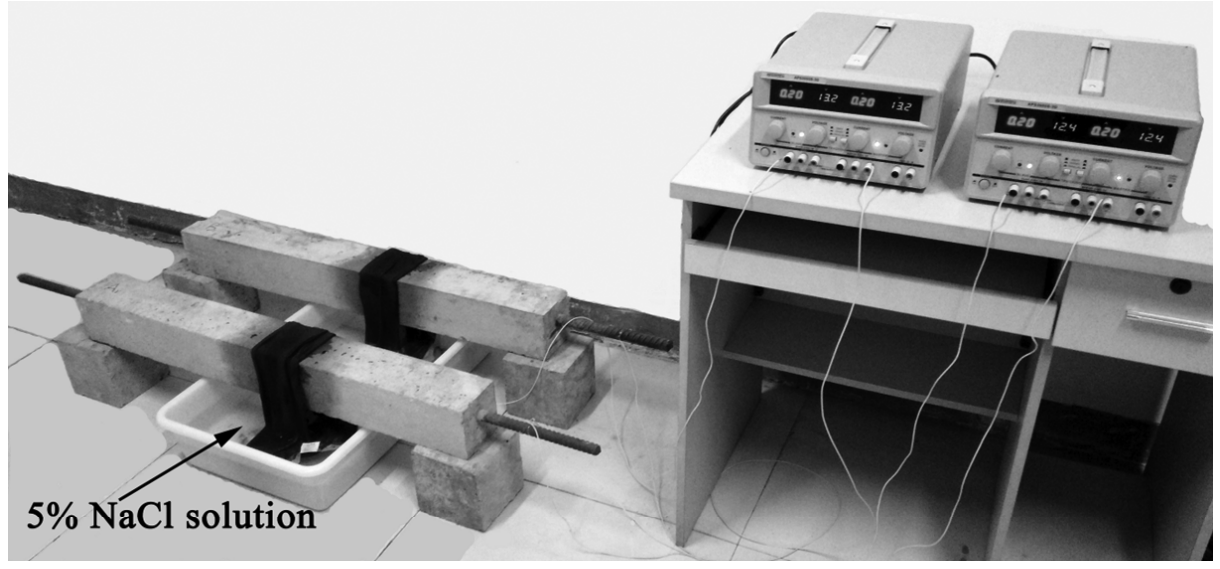

Figure 3. The accelerated galvanic corrosion test of the specimens.

position coordinates $(\boldsymbol{X}, \boldsymbol{Y}$ and $\boldsymbol{Z})$ by the ethernet interface of the ESC.

\subsection{Automation Software System}

An automatical scanning and measuring software, running on a computer, is developed by the $\mathrm{C} \#$ programming language for the automatic test, and the software operation interface is illustrated in Fig. 2. A series of rules generating the automatic scanning and measuring commands are selfdefined in the program. The rules comprise the fields of the "Checked", "X Destination", "Y Destination", "Z Destination", "X Speed", "Y Speed", "Z Speed", "Save Data" and "Delay". The data acquisition frequency can reach up to $10 \mathrm{~Hz}$. The software is capable of real-time showing measuring data and saving them in a text file and SQLite database simultaneously. Once all the measuring tasks are completed, this software can send an e-mail to the experimenters whose e-mail addressed have scheduled in the software settings.

\section{Experiment Details}

\subsection{Accelerated Galvanic Corrosion of Specimens}

Two similar RC specimens were prepared for corrosion experiment and MMM testing. Each specimen was a rectangular concrete block in which an HRB335 reinforcement bar was inserted. The diameter and the length of the rebar were $25 \mathrm{~mm}$ and $150 \mathrm{~cm}$, respectively. The dimensions of the two concrete blocks were $100 \mathrm{~cm} \times 10.5 \mathrm{~cm} \times 12.5 \mathrm{~cm}$. The concrete grade and cover thickness of each specimen were $\mathrm{C} 30$ and $3 \mathrm{~cm}$, respectively. These specimens were corroded under a current intensity of $0.2 \mathrm{~A}$ in the fixed position based on an accelerated galvanic corrosion method illustrated in Fig. 3. One end of the rebar was connected 


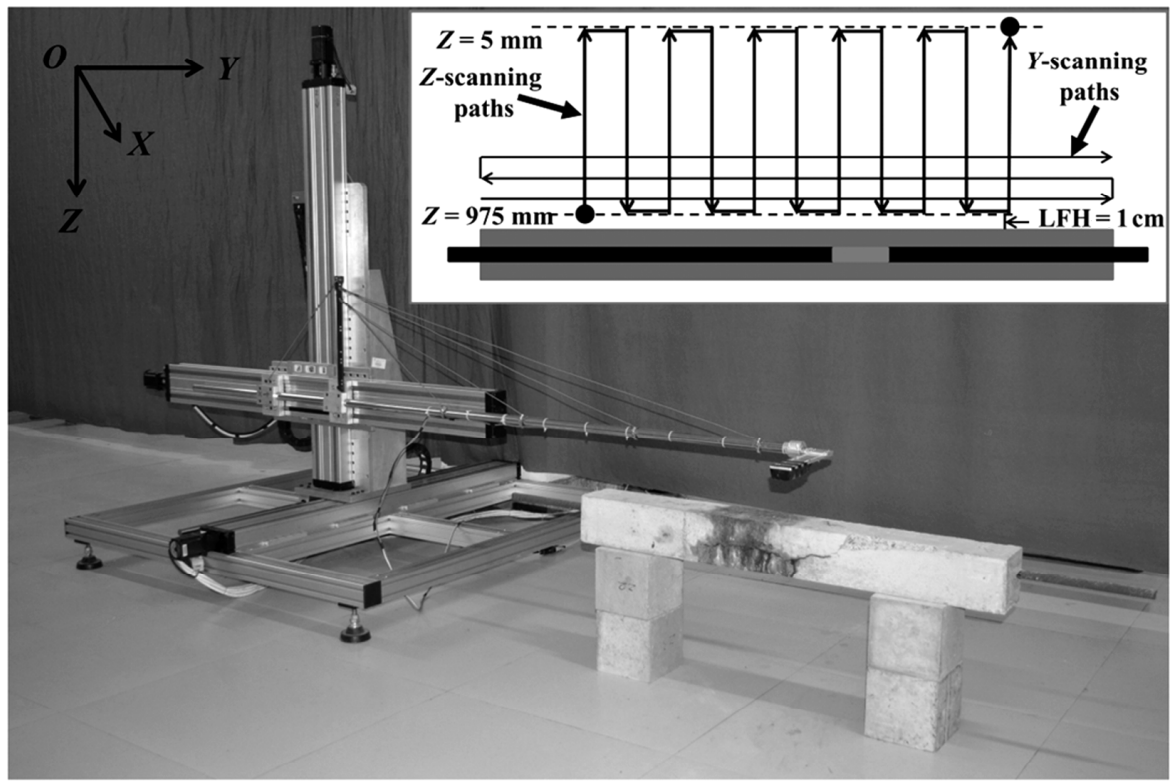

Figure 4 . The 3D automatic scanning and measuring system in operation.

with the positive electrode of the power supply via wires, while the auxiliary electrode was connected to the negative electrode. The electrolyte of $5 \% \mathrm{NaCl}$ solution was prepared in advance, and the towel was soaked above the auxiliary electrode in the electrolyte. In this way, the electrolyte could pass the towel into the concrete based on the siphon effect, and the internal rebar of concrete could be corroded at a specified location. The corrosion level was controlled by the corroded current and time according to the Faraday law, and the two RC samples were corroded under a current intensity of $0.2 \mathrm{~A}$ and the corrosion time is 4, 7 and 9 days, respectively. The preconceived corrosion length of each specimen was $20 \mathrm{~cm}$.

\subsection{Scanning and Measuring Test}

The scanning paths and methods of the magnetometer are illustrated in Fig. 4, where $\boldsymbol{B}_{\boldsymbol{y}}$ is defined as a tangential component of the magnetic flux density for the specimens. The distance between the bottom of the sensor and the top of the specimen is defined as the lift-off height (LFH). The corrosion region was in the range of $Y=600-800 \mathrm{~mm}$. The initial LFH of the magnetometer is $\sim 1 \mathrm{~cm}$ (where $Z=975 \mathrm{~mm}$ ). The magnetometer was moved at a speed of $5.0 \mathrm{~cm} / \mathrm{min}$, and all the test data, including $\boldsymbol{X}, \boldsymbol{Y}, \boldsymbol{Z}, \boldsymbol{B}_{\boldsymbol{x}}$, $\boldsymbol{B}_{\boldsymbol{y}}$, and $\boldsymbol{B}_{\boldsymbol{z}}$ components, were automatically acquired and recorded with a frequency of $2 \mathrm{~Hz}$.

\section{Experimental Results and Discussions}

The results of the two RC specimens share similar tendency, so just data from one representative specimen are addressed in this paper.

Before the specimen was corroded, we scanned and measured the magnetic field of the sample along the $Y$ direction at the $\mathrm{LFH}$ of 1,2 and $3 \mathrm{~cm}$, respectively, and obtained the $\boldsymbol{B}_{\boldsymbol{y}}-\boldsymbol{Y}$ curves as shown in Fig. 5(a). In the same way, we obtained the $\boldsymbol{B}_{y}-\boldsymbol{Y}$ curves corroded for 4,7 and 9 days as shown in Figs. 6(b), (c) and (d), respectively. Figure 5 shows that the curves with different LFHs are almost parallel to each other in the intact condition, while the curves with different LFHs intersect at two points when the sample is corroded for 4, 7 and 9 days, respectively. The distances between the two intersecting points are 130, 156 and $168 \mathrm{~mm}$ for the different corrosion time, respectively; these distances are all less than $20 \mathrm{~cm}(Y=600-800 \mathrm{~mm})$. The result indicates that: the longer the corrosion period is, the closer the distance is to the real length of the corrosion region; the smaller the LFH is, the bigger the absolute value of the extrema of $\boldsymbol{B}_{y}$ in corrosion area is, namely, the stronger the SMFL signal is; the position and region of corrosion can be estimated qualitatively by analysing the position and interval of the two intersecting points, respectively. These experimental phenomena have a phenomenological model referred to [5].

We scanned and measured the magnetic field of the specimen according to the $Z$-scanning paths illustrated in Fig. 4 and obtained the $\boldsymbol{B}_{\boldsymbol{y}}-\boldsymbol{Z}$ curves at different $\mathrm{Y}$ positions of the specimen corroded for 4 days as shown in Fig. 6. A difference observed from Fig. 6 demonstrated that the tangential component values of the magnetic flux density, namely $\boldsymbol{B}_{y}$, approach to a certain value with the increase in the LFH at the non-corroded positions such as $Y=20 \mathrm{~mm}$ and $Y=900 \mathrm{~mm}$, however at the corroded positions, i.e. $Y=620 \mathrm{~mm}, Y=640 \mathrm{~mm}, Y=700 \mathrm{~mm}$ and $Y=740 \mathrm{~mm}$, the signals of $\boldsymbol{B}_{y}$ appear a peak with the increase in the LFH. The peak point is defined as the reversal point in this paper. Because there is not any reversal point in the non-corroded areas, the $Z$ values of the initial scanning point are regards as the $Z$ values of reversal points for plotting curves conveniently.

Figure 7 shows the $Z$ values of reversal points in the different positions of the specimen for various corrosion period. The experimental corrosion area was the region of 


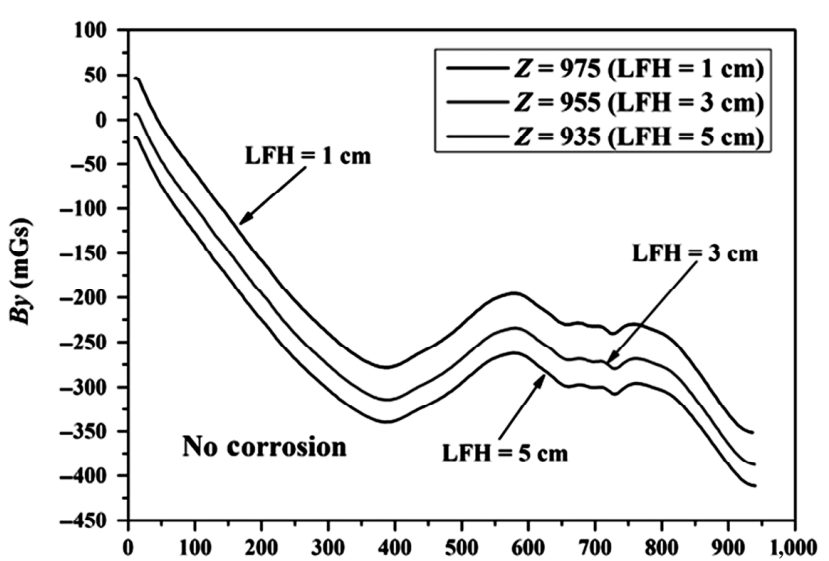

(a)

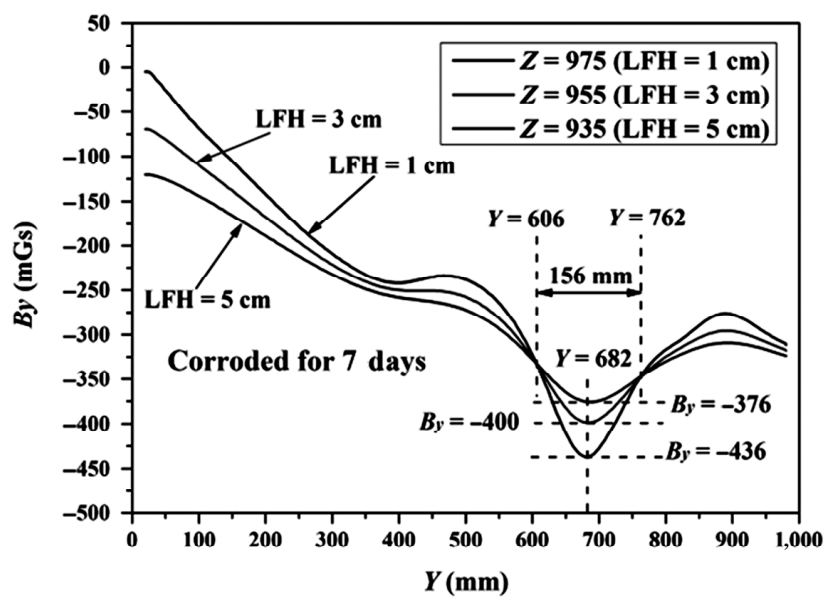

(c)

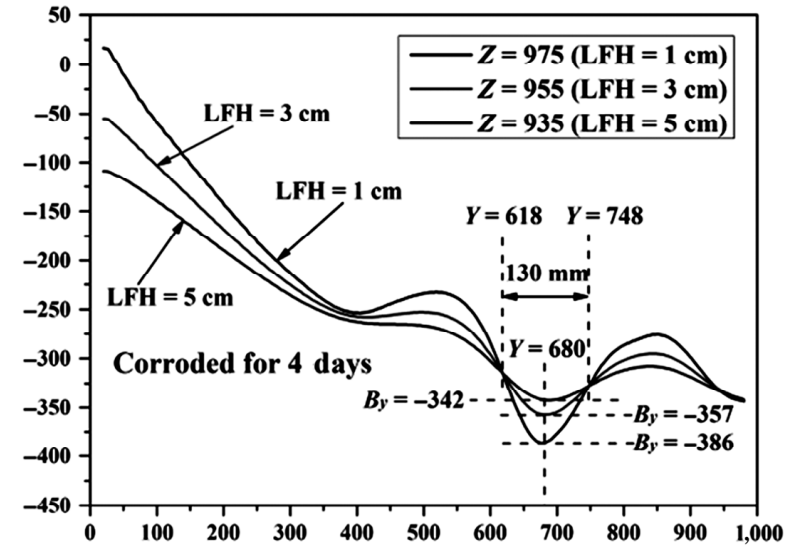

(b)

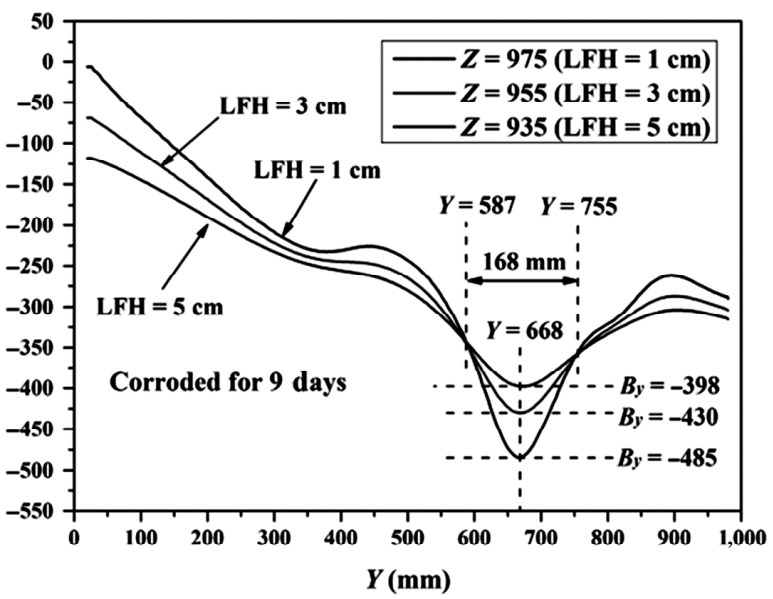

(d)

Figure 5. The $\boldsymbol{B}_{y}-\boldsymbol{Y}$ curves at different lift-off heights of the magnetometer: (a) no corrosion; (b) corroded for 4 days; (c) corroded for 7 days; and (d) corroded for 9 days.

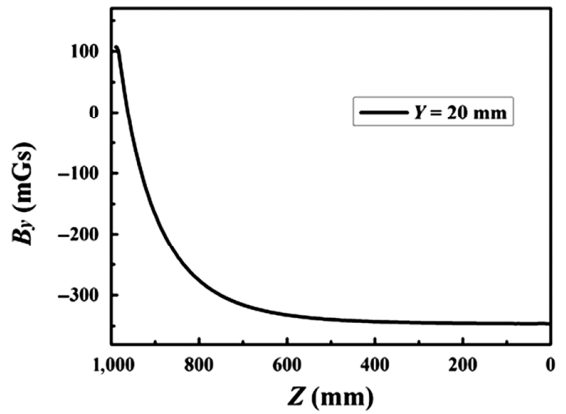

(a)

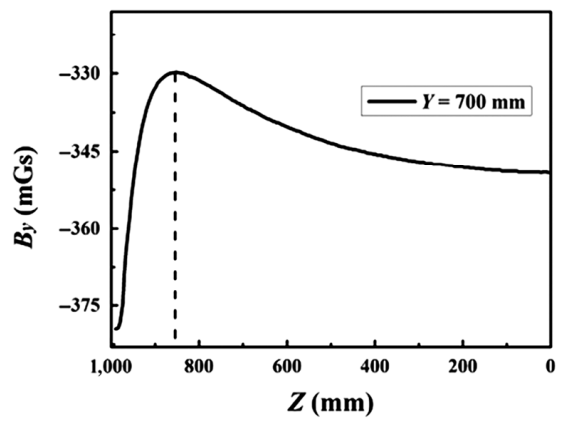

(d)

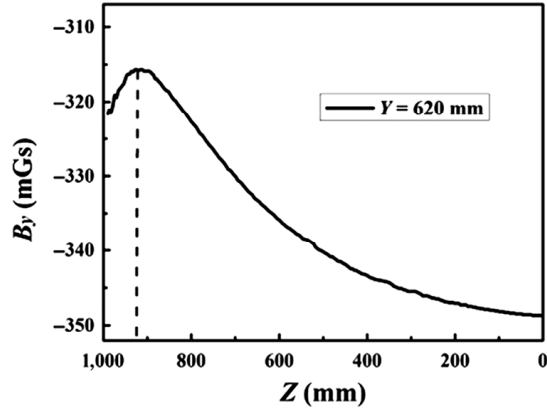

(b)

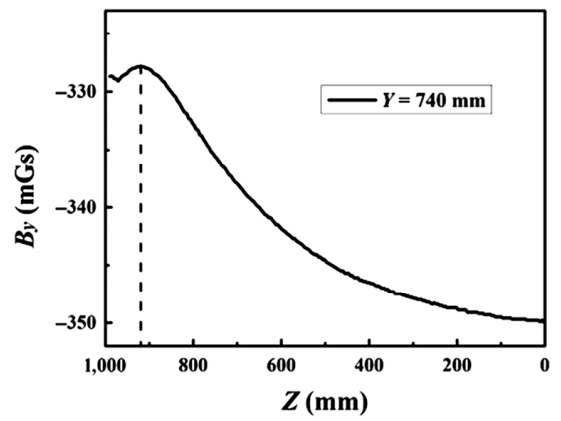

(e)

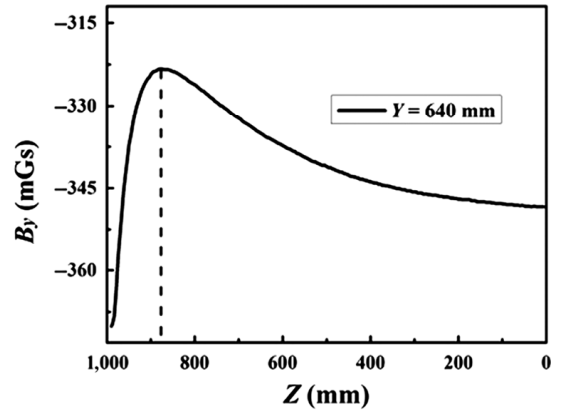

(c)

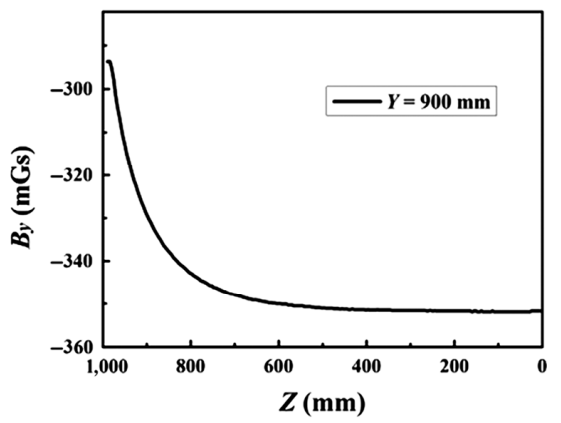

(f)

Figure 6. The $\boldsymbol{B}_{y}-\boldsymbol{Z}$ curves at different $Y$ positions corroded for 4 days. The vertical dashed line indicates the position of the reversal point. 


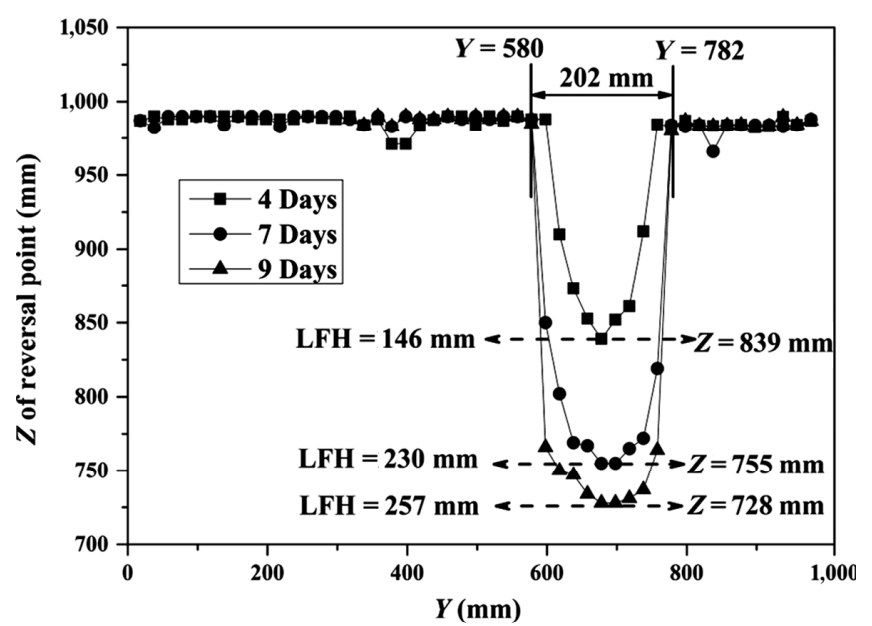

Figure 7 . The $Z$ values of reversal points in the different positions of the specimen for different corrosion time.

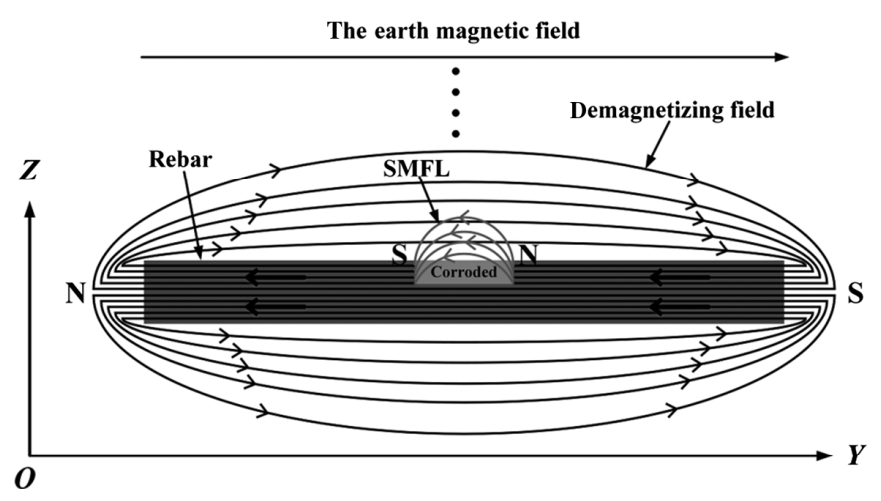

Figure 8. Schematic diagrams of the magnetic field distribution around the corroded rebar bar.

$Y=600-800 \mathrm{~mm}$, but the actual corrosion area is the part of $Y=580-782 \mathrm{~mm}$, because of the electrolyte, absorbed by the towel, soaked into the concrete randomly. Namely, the corrosion does not occur in an intentional way. Figure 7 shows that the more severe the corrosion degree is, the smaller the $Z$ of reversal point is, namely, the higher the $\mathrm{LFH}$ of the reversal point is.

It is well known that a weak magnetic field exists near the surface of the Earth generated by the planet itself. It can be seen as a stable and uniform magnetic field in a relatively small space compared with the Earth. When a ferromagnetic material is put into the magnetic field, the ferromagnetic materials will be magnetized so that it can be treated as a magnet. Rebar is a typical ferromagnetic material, and it has a higher magnetic permeability than it in air or concrete. Figure 8 shows that higher magnetic flux density passes through the rebar when the rebar has not been corroded. The magnetic flux density, detected by the magnetometer, is the vector superposition of the Earth magnetic field and the demagnetizing field of the magnet. The demagnetizing field of the magnet plays a dominating role near the surface of the magnet when the rebar has not been corroded, so the tangential component value of the magnetic flux density approaches to a certain value with the increase in the LFH at the non-corroded positions, and the certain value approximates to the tangential component of the Earth magnetic field. However, once the rebar has been corroded to a certain degree, a magnetic flux leakage signal will be generated from the corroded position due to the high magnetic permeability gradient in the vicinity of the corrosion area, so it is the so-called self-magnetic flux leakage. The direction of SMFL is opposite to the orientation of the demagnetizing field as shown in Fig. 8. Hence, there is a competition between the demagnetizing field and the SMFL above the corrosion area. The SMFL dominates near the rebar, and the demagnetizing field is gradually increasing with larger of the LFH of the sensor. When the demagnetizing field intensity is larger than the SMFL at a certain LFH, the data reversal phenomenon appears. On the one hand, the more severe the damage is, the larger the SMFL is. As a result, the total magnetic flux density is decreasing. On the other hand, the SMFL influences the wider space with a larger degree of the corrosion, so the LFH value of the reversal point is higher. Thus, the LFH value of the reversal point can serve as a judging criterion to assess the corrosion degree of the rebar qualitatively, and then the corrosion level of the RC can be obtained.

\section{Conclusion}

In this work, a 3D scanning and measuring device and its software are the essential tools for experimental study. The proposed 3D system not only can accurately acquire the spatial position and corresponding 3D magnetic flux density but also can be regarded as a high-precision 3D scanning platform for further study. Therefore, we will install new sensors on the device in the future.

The corrosion region of rebar in concrete can be qualitatively estimated as follows. Firstly, scan and measure the magnetic field of the specimen along the $Y$ direction at the two or more LFHs. Secondly, plot all the $\boldsymbol{B}_{\boldsymbol{y}}-\boldsymbol{Y}$ curves in one figure and then investigate whether or not there are two intersecting points. Thirdly, if these points are identified, their positions and the distance between them imply the presence of the corrosion. Namely, the more severe the corrosion is, the closer the distance is to the length of corrosion region.

The corrosion degree of rebar in concrete can be semiquantitatively assessed as follows. Firstly, scan and measure the magnetic field of the specimen along the $Z$ direction at different corroded positions. Secondly, plot the $\boldsymbol{B}_{\boldsymbol{y}}-\boldsymbol{Z}$ curves and then investigate whether or not have an extreme value varying with the LFHs of the magnetic sensor. Thirdly, assess the corrosion degree by the LFH of the reversal point semi-quantitatively. The assessment standard is that the more severe the corrosion degree is, the higher the LFH of the reversal point is.

This work is a significative exploration for rebar corrosion detection, but some experimental factors have not been fully considered yet, including the pressure induced by the corrosion expansion of the rebar, the physical parameters of the rebar, the strength of concrete, the coupling of two or more rebars, etc. They are addressed in the future. 


\section{Acknowledgement}

This work was supported by the National Science Fund for Distinguished Young Scholars (51425801), the National Key Research and Development Program of China (2016YFC0802202, 2017YFC0806007), the National Natural Science Foundation of China (51278512, 51508058, 11404045), the Social Livelihood Science and Technology Innovation Special of Chongqing (cstc2015shmszx30012), the Science and Technology Project of Guizhou Provincial Transportation Department (2016-123-006), the Science and Technology Planning Project of Yunnan Province (2017IB025), the Science and Technology Research Project of Chongqing Education Commission (KJ1710262, KJ1500528), and the Graduate Student Research Innovation Project in Chongqing (CYB16178, CYS17194).

\section{References}

[1] A. Zaki, H.K. Chai, D.G. Aggelis, and N. Alver, Nondestructive evaluation for corrosion monitoring in concrete: A review and capability of acoustic emission technique, Sensors, 15(8), 2015, 19069-19101.

[2] I. Fernandez, J.M. Bairán, and A.R. Marí. Corrosion effects on the mechanical properties of reinforcing steel bars. Fatigue and $\sigma-\varepsilon$ behavior, Construction and Building Materials, 101, $2015,772-783$.

[3] Y.C. Ou and N.D. Nguyen. Influences of location of reinforcement corrosion on seismic performance of corroded reinforced concrete beams, Engineering Structures, 126, 2016, 210-223.

[4] H.W. Song and V. Saraswathy, Corrosion monitoring of reinforced concrete structures - a review, International Journal of Electrochemical Science, 2, 2007, 1-28.

[5] H. Zhang, L. Liao, R.Q. Zhao, et al., The non-destructive test of steel corrosion in reinforced concrete bridges using a micro-magnetic sensor, Sensors, 16(9), 2016, 1439.

[6] D.A. Aikens, Electrochemical methods, fundamentals and applications, Journal of Chemical Education, 60(1), 1983, A25.

[7] Z.S. Chen, J.T. Zhou, K.T. Tse, et al., Alignment control for a long span urban rail-transit cable-stayed bridge considering dynamic train loads, Science China Technological Sciences, 59(11), 2016, 1759-1770.

[8] Z.D. Wang, Y. Gu, and Y.S. Wang, A review of three magnetic NDT technologies, Journal of Magnetism and Magnetic Materials, 324(4), 2012, 382-388.

[9] H.G. Ramos and A.L. Ribeiro, Present and future impact of magnetic sensors in nde, Procedia Engineering, 86, 2014, 406-419.

[10] Y. Takahashi, E. Matsubara, S. Suzuki, et al., In-situ X-ray diffraction of corrosion products formed on iron surfaces, Materials Transactions, 46(3), 2005, 637-642.

[11] K. Kobayashi and N. Banthia, Corrosion detection in reinforced concrete using induction heating and infrared thermography, Journal of Civil Structural Health Monitoring, 1(1-2), 2011, $25-35$.

[12] A. Nair and C.S. Cai, Acoustic emission monitoring of bridges: Review and case studies, Engineering Structures, 32(6), 2010, 1704-1714.

[13] T. Ren, Q.Y. Liu, Y.H. Chen, and S.H. Ji, Variable pitch helical drive in-pipe robot, International Journal of Robotics and Automation, 31(3), 2016, 263-271.

[14] A.A. Dubov, A study of metal properties using the method of magnetic memory, Metal Science and Heat Treatment, 39(9), 1997, 401-405.

[15] K. Yao., B. Deng, and Z.D. Wang, Numerical studies to signal characteristics with the metal magnetic memory-effect in plastically deformed samples, NDT $\&$ E International, 47(4), $2012,7-17$.

[16] Z.M. Zhou, T.H. Shi, R.Q. Yu, et al., Metal magnetic memorybased fluxgate sensor for imaging detection of coiled tubing, Insight, 56(12), 2014, 691-694.
[17] K. Yao, Z.D. Wang, B. Deng, and K. Shen, Experimental research on metal magnetic memory method, Experimental Mechanics, 52(3), 2012, 305-314.

[18] R. Kaushik, J.Z. Xiao, S.L. Joseph, and W. Morris, Polygonbased 3D scan registration with dual-robots in structured indoor environments, International Journal of Robotics and Automation, 27(1), 2012, 101.

[19] J. Zhang, F.C. Tian, S.X. Yang, et al., An inteligent and automatic control method for tobacco flue-curing based on machine learning, International Journal of Robotics and Automation, 31(6) 2016, 509-518.

\section{Biographies}

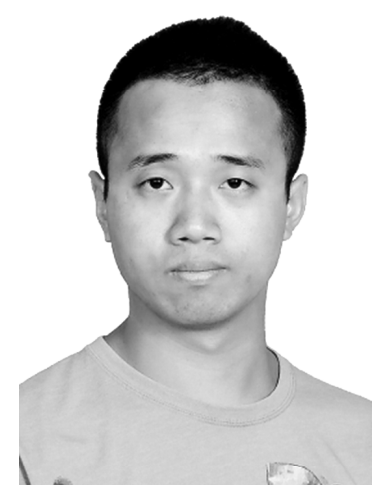

Hong Zhang is currently studying for a doctor's degree in Bridge and Tunnel Engineering at Chongqing Jiaotong University of China. His research interests include system integration, structural health monitoring and non-destructive inspection.

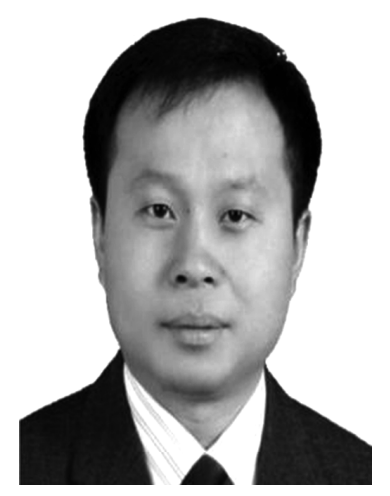

Jianting Zhou received his Ph.D. degree in Intelligent Structure from the University of Chongqing, China, in 2007. He is a full professor and doctoral advisor of Chongqing Jiaotong University. His research interests include bridge health monitoring and structure safety evaluation.

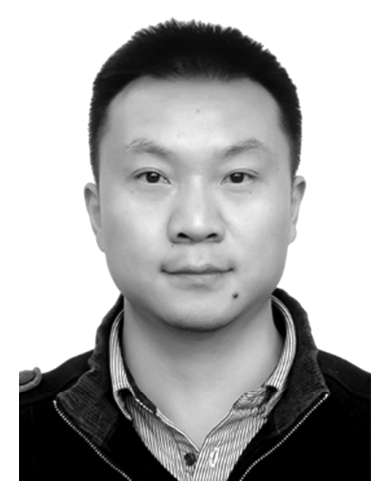

Ruiqiang Zhao received his Ph.D. degree in Optics from the Institute of Physics, Chinese Academy of Sciences, in 2014. His research interest includes the engineering application of the physical method.

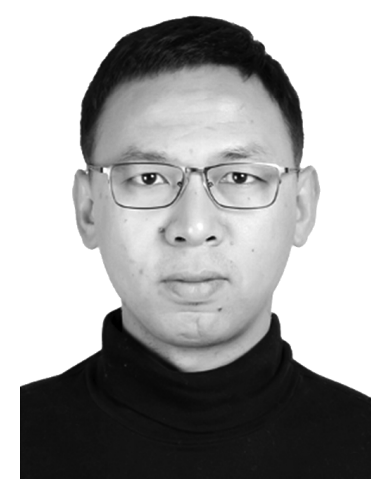

Leng Liao received his Ph.D. degree in Condensed Matter Physics from Institute of Physics, Chinese Academy of Science, in 2006. His research interests include applied electromagnetics, innovative physical sensors and nondestructive test techniques in civil engineering. 


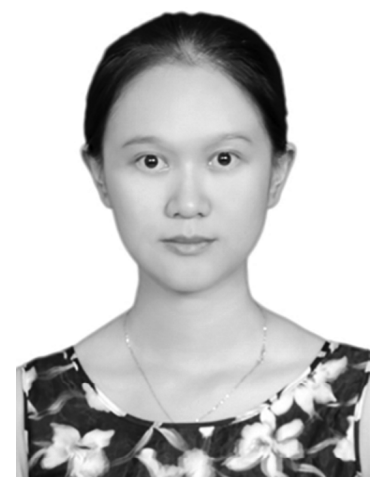

Mao Yang received her master degree at the College of Civil Engineering, Chongqing Jiaotong University in 2016. Her main research interests include the bridge nondestructive inspection and bridge health monitoring.

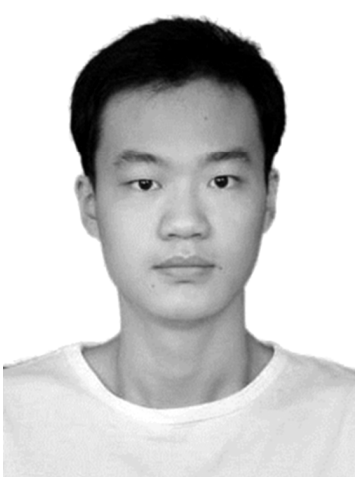

Runchuan Xia is currently studying for a doctor's degree in Bridge and Tunnel Engineering at Chongqing Jiaotong University. His research interests mainly include the non-destructive inspection and structural health monitoring. 